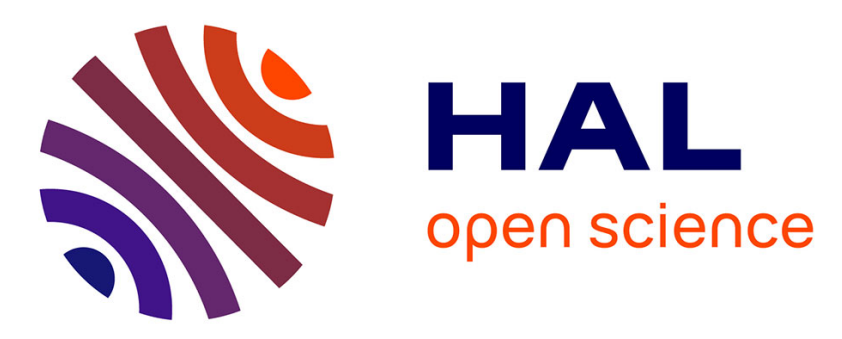

\title{
Bayesian probabilistic analysis of a nuclear power plant small loss of coolant event tree model with possibilistic parameters
}

Chung-Kung Lo, Nicola Pedroni, Enrico Zio

\section{- To cite this version:}

Chung-Kung Lo, Nicola Pedroni, Enrico Zio. Bayesian probabilistic analysis of a nuclear power plant small loss of coolant event tree model with possibilistic parameters. Safety, Reliability and Risk Analysis, Beyond the Horizon, Proceedings of the European Safety and RELiability Conference (ESREL) 2013, 2013, Amsterdam, Netherlands. pp.3321-3328, 10.1201/b15938-505 . hal-01104385

\section{HAL Id: hal-01104385 \\ https://hal-centralesupelec.archives-ouvertes.fr/hal-01104385}

Submitted on 16 Jan 2015

HAL is a multi-disciplinary open access archive for the deposit and dissemination of scientific research documents, whether they are published or not. The documents may come from teaching and research institutions in France or abroad, or from public or private research centers.
L'archive ouverte pluridisciplinaire HAL, est destinée au dépôt et à la diffusion de documents scientifiques de niveau recherche, publiés ou non, émanant des établissements d'enseignement et de recherche français ou étrangers, des laboratoires publics ou privés. 
C.-K. Lo, N. Pedroni, E. Zio, "Bayesian probabilistic analysis of a nuclear power plant small loss of coolant event tree model with possibilistic parameters", in: R.D.J.M. Steenbergen, P.H.A.J.M. van Gelder, S. Miraglia and A. C.W.M. Ton. Vrouwenvelder (Eds.), Safety, Reliability and Risk Analysis, Beyond the Horizon, Proceedings of the European Safety and RELiability Conference (ESREL) 2013, Amsterdam, The Netherlands, 29 September-2 October 2013, pp. 3321-3328, Taylor and Francis Group, London, UK, 2014, ISBN 978-1138001237. 


\title{
Bayesian probabilistic analysis of a nuclear power plant small loss of coolant event tree model with possibilistic parameters
}

\author{
Chung-Kung Lo ${ }^{a}$, N. Pedroni ${ }^{a}$, E. Zio ${ }^{a, b}$ \\ ${ }^{a}$ Chair on Systems Science and the Energetic Challenge, European Foundation for New Energy - Electricité \\ de France, at École Centrale Paris - Supelec, France \\ ${ }^{b}$ Department of Energy, Politecnico di Milano, Italy
}

\begin{abstract}
Nuclear Power plant risk analysis models (e.g., Fault and Event Trees) contain parameters (e.g., probabilities) that are epistemically uncertain, i.e., known with poor precision. In current Probabilistic Risk Assessment (PRA) practice, epistemic uncertainty is described by predetermined probability distributions. However, a probabilistic representation of epistemic uncertainty is difficult to justify in those cases in which the analysis is carried out based on imprecise and incomplete information. In this paper, we describe epistemic uncertainty by possibility distributions, which encode families of probability distributions and, thus, represent the analyst's imprecise knowledge of the uncertain model parameters. This work addresses the issue of updating, in a Bayesian framework, the possibilistic representation of the epistemically-uncertain parameters of risk models as new information (e.g., data) becomes available: a purely possibilistic counterpart of the classical, well-grounded probabilistic Bayes theorem is adopted. The method is applied to revise, by means of real plant failure data, the possibility distributions describing the epistemic uncertainties in the probabilities of occurrence of accident sequences following a small loss of coolant event in a nuclear power plant.
\end{abstract}

\section{INTRODUCTION}

In nuclear power plants (NPP), Probabilistic Risk Assessment (PRA) is used to evaluate accident risks in terms of consequences and probabilities. The assessment of system failure probabilities is done by means of event tree analysis (ETA) and fault tree analysis (FTA). The risk indicators computed by PRA play an important role for safety decision making. Then, the calculation of these indicators must be robust with respect to uncertainties.

In general, the uncertainties in a NPP's PRA models can be categorized as either aleatory or epistemic. Aleatory uncertainty reflects our inability to predict the random nature of the basic events considered, whereas epistemic uncertainty represents our lack of knowledge of the values of the parameters used to describe the basic events (e.g. probabilities, failure rates...). Many recent researches have shown that the precision of final results is mainly affected by epistemic uncertainty (Drouin $M$ et al. 2010). Epistemic uncertainty can be roughly split into three categories: parameter, model and completeness. In this paper, we focus on the parameter uncertainty only.

In the traditional PRA practice, the epistemic uncertainty in the parameters related to basic events occurrence is described by using a presumed probability distribution, e.g. lognormal, Gamma, or Beta. The choice of distribution is mainly done on the basis of expert judgment and conventional reasons, e.g. conjugate Gamma and Poisson, Beta and Binominal distributions. Many recent studies (Baudrit C et al. 2007, Durga Rao K et al. 2007) concluded that in case of insufficient data to justify the choice of a specific distribution form, it is more appropriate to use a family of probability distributions for representing the imprecise and incomplete information. Such a family can be defined by probability bounds ( $\mathrm{p}$-box), possibility distributions, or belief and plausibility functions within the Dempster-Shafer Theory (DST) of evidence: a framework using belief and plausibility functions for uncertainty analysis has been illustrated through a practical example for NPP PRA in (Le Duy T.D et al. 2013).

In the context of NPP PRA, the traditional decision making process within the probabilistic approach relies on the comparison of risk indicators to a safety threshold. The uncertainty bound of the risk indicator usually makes it difficult to make a decision. How to narrow down the uncertainty bound is always an important issue. For a single probability density function, the Bayes' rule is commonly used to reduce the uncertainty bound with new information such as plant specific data. Although using non-probabilistic frameworks (i.e. families of probability distributions) for uncertainty analysis provides more realistic and robust results, it indeed expands the uncertainty bounds of the risk indicator. We, then, need to know how to directly use the Bayes' rule to revise a family of probability distributions.

In this paper, we use possibility distributions to describe the epistemic uncertainty in the parameters of risk models (i.e. the probabilities of events in Fault and Event trees) and we address the issue of 
updating, in a Bayesian framework, this possibilistic representation of epistemic uncertainty by means of newly available data. The main purpose of this paper is to demonstrate how to describe epistemic uncertainty by possibility distributions and embed the possibilistic Bayesian updating process into an uncertainty analysis framework.

This paper is organized as follows. In section 2, we recall the main non-probabilistic approaches used in the literature to represent the epistemic uncertainty (with particular emphasis on possibility theory) and compare them. In section 3, we introduce the process of Bayesian update of possibility distributions when new information is available. Section 4 presents the study case and the uncertainty propagation results. Finally, some conclusions are drawn in Section 5.

\section{NON-PROBABILISTIC REPRESENTATIONS OF EPISTEMIC UNCERTAINTY}

Suppose $F_{u}$ and $F_{l}$ are non-decreasing functions from the real line $R$ into $[0,1]$ and $F_{l}(x) \leq F_{u}(x)$ for all $x \in R$. Let $\left[F_{u}, F_{l}\right]$ denote the set of all nondecreasing function $F$ from the reals into $[0,1]$ such that $F_{l}(x) \leq F(x) \leq F_{u}(x)$. When the functions $F_{u}$ and $F_{l}$ circumscribe an imprecisely known probability distribution, we call $\left[F_{u}, F_{l}\right]$, specified by the pair of functions, a "probability box" or "p-box" for that distribution. For an epistemically-uncertain parameter $X$ whose distribution $F$ is unknown except that it is within the p-box, then $F_{l}(x)$ is a lower bound on $F(x)=P(X \leq x)$ which is the (imprecisely known) probability that the uncertain parameter $X$ is smaller than $x$. Likewise, $F_{u}(x)$ is an upper bound on the same probability.

In the subsections below, we showed how to represent such imprecise distributions using Dempster-Shafer structures and possibility distributions.

\subsection{Dempster Shafer Theory of Evidence}

The Dempster-Shafer theory (Shafer G. 1976) is a mixed representation, which combines the probabilistic and the interval representations in a single representation. DST over the set of the real numbers resembles to describe probability theory except that the locations at which the probability mass resides are sets of real values, rather than precise points. These sets associated with non-null mass are called focal elements. The probability mass, noted $m$, associated with a given set can be understood as the weight of evidence that truth is in that set. The uncertainty associated to an epistemic parameter $X$ on the real line $R$ can be represented by the so-called Dempster-Shafer structure as: $\left\{\left(\left[a_{1}, b_{1}\right], m_{1}\right),\left(\left[a_{2}, b_{2}\right], m_{2}\right), \ldots,\left(\left[a_{n}, b_{n}\right], m_{n}\right)\right\}$

where $a_{i} \leq b_{i}, \sum_{i=1}^{n} m_{i}\left(\left[a_{i}, b_{i}\right]\right)=1$ and $\left[a_{i}, b_{i}\right] \subseteq R$ for all $1 \leq i \leq n$. The Dempster-Shafer structure is thus a collection of pairs consisting of closed intervals and corresponding mass.

The belief function of the uncertain parameter $X$ belonging to a subset $A \subseteq R$ is the sum of all masses associated with sets that are subsets of $A$. Thus,

$$
\operatorname{Bel}(X \in A)=\sum_{\left[a_{i}, b_{i}\right] \subseteq A} m_{i}
$$

The plausibility function is the sum of all masses associated with sets that overlap with or merely touch the set $A$ so that

$$
P l(X \in A)=\sum_{\left[a_{i}, b_{i}\right] \cap A \neq \Phi} m_{i}
$$

Clearly, $\operatorname{Bel}(X \in A) \leq P l(X \in A)$ and given a Dempster-Shafer structure as in equation (1), the lower and upper bounds of the probability functions are equated with the belief and plausibility functions (Walley P. 1991, Dempster A. 1967) defined as:

$$
\begin{aligned}
& F_{l}(x)=\operatorname{Bel}(X \in[-\infty, x])=\sum_{b_{i} \leq x, i=1}^{n} m_{i} \\
& F_{u}(x)=P l(X \in[-\infty, x])=\sum_{a_{i} \leq x, i=1}^{n} m_{i}
\end{aligned}
$$

\subsection{Numerical possibility theory}

Possibility theory (Dubois D et al. 2000) is relevant to represent consonant imprecise knowledge. The basic notion is the possibility distribution, denoted $\pi$, an upper semi-continuous mapping from the real line to the unit interval. A possibility distribution describes the more or less plausible values of some uncertain parameter $X$. Possibility theory provides two evaluations of the likelihood of an event, for instance whether the value of a real variable $X$ does lie within a certain interval: the possibility $\Pi$ and the necessity $N$ are defined as

$$
\begin{aligned}
& \Pi(A)=\sup _{x \in A} \pi(x) \\
& N(A)=1-\Pi(\bar{A})=\inf _{x \notin A}(1-\pi(x))
\end{aligned}
$$

A unimodal numerical possibility distribution may also be viewed as a nested set of confidence intervals, which are the $\alpha$-cuts $\left[\underline{x}_{\alpha}, \bar{x}_{\alpha}\right]=$ 
$\{x, \pi(x) \geq \alpha\}$ of $\pi$. The degree of certainty that $\left[\underline{x}_{\alpha}\right.$, $\left.\bar{x}_{\alpha}\right]$ contains $X$ is $N\left(\left[\underline{x}_{\alpha}, \bar{x}_{\alpha}\right]\right)=1-\alpha$ (if $\pi$ is continuous). Conversely, a nested set of intervals $A_{i}$ with degrees of certainty $\lambda_{i}$ that $A_{i}$ contains $X$ is equivalent to the possibility distribution

$$
\pi(x)=\min _{i=1 \ldots n}\left\{1-\lambda_{i}, x \in A_{i}\right\}
$$

provided that $\lambda_{i}$ is interpreted as a lower bound on $N\left(A_{i}\right)$, and $\pi$ is chosen as the least specific possibility distribution satisfying these inequalities (Dubois D. \& Prade H. 1992).

We can interpret any pair of dual functions necessity/possibility $[N, \Pi]$ as upper and lower probabilities induced from specific probability families.

Let $\pi$ be a possibility distribution inducing a pair of functions $[N, \Pi]$. We define the probability family $\mathrm{P}(\pi)=\{p, \forall A$ measurable, $N(A) \leq p(A)\}=\{p, \forall A$ measurable, $p(A) \leq \Pi(A)\}$. In this case, $\sup _{p \in \mathrm{P}(\pi)} p(A)=\Pi(A) \quad$ and $\quad \inf _{p \in \mathrm{P}(\pi)} p(A)=N(A)$ hold. In other words, the family $\mathrm{P}(\pi)$ is entirely determined by the probability intervals it generates.

Suppose pairs (interval $A_{i}$, necessity weight $\lambda_{i}$ ) supplied by an expert are interpreted as stating that the probability $p\left(A_{i}\right)$ is at least equal to $\lambda_{i}$ where $A_{i}$ is a measurable set. We define the probability family as follows: $\mathrm{P}(\pi)=\left\{p, \forall A_{i} \quad \lambda_{i} \leq p(A)\right\}$. We thus know that $\bar{p}=\Pi$ and $\underline{p}=N$.

\subsection{Relationship between belief and plausibility functions and possibility distributions}

For a unimodal continuous possibility $\pi$ with core $\{a\}$ (i.e. $\Pi(\{a\})=\pi(a)=1$ and $\forall x \neq a, \pi(x) \neq 1)$, the set of probability measures $\mathrm{P}(\pi)$ can be more conveniently described by a condition on the cumulative distribution functions of these probabilities, that is $\mathrm{P}(\pi)=\{p, \forall x, y, x \leq a \leq y, F(x)$ $+1-F(y) \leq \max (\pi(x), \pi(y))\}$. Note that we can choose $x$ and $y$ such that $\pi(x)=\pi(y)$ in the expression of $\mathrm{P}(\pi)$, i.e. suppose that $[x, y]$ is a cut of $\pi$. If $I_{\alpha}$ is the $\alpha$-cut of $\pi$, it holds that $\mathrm{P}(\pi)=\left\{p, p\left(I_{\alpha}\right)\right.$ $\left.\geq N\left(I_{\alpha}\right), \forall \alpha \in(0,1]\right\}$.

Define a particular probability box $\left[F_{l}, F_{u}\right]$ such that $F_{u}(x)=\Pi(X \in(-\infty, x])$ and $F_{l}(x)=N(X \in(-\infty$, $x])$. It is clear that

$$
F_{u}(x)=\left\{\begin{array}{cc}
\pi(x) & \text { for } x \leq a \\
1 & \text { for } x \geq a
\end{array}\right.
$$

$$
F_{l}(x)=\left\{\begin{array}{cc}
0 & \text { for } x \leq a \\
1-\pi(x) & \text { for } x \geq a
\end{array}\right.
$$

The probability box $\left[F_{l}, F_{u}\right]$ above has an important specific feature: there exists a real value $a$ such that $F_{u}(a)=1$ and $F_{l}(a)=0$. It means that the p-box contains the deterministic value $a$, so that the two cumulative distributions are acting in disjoint areas of the real line separated by this value. We can retrieve a possibility distribution from such two cumulative distribution functions as $\pi=\min \left(F_{u}, 1-\right.$ $\left.F_{l}\right)$ and, thus, retrieve the possibility distribution that generated the $\mathrm{p}$-box.

\section{BAYESIAN UPDATE PROCESS OF THE EPISTEMICALLY-UNCERTAIN PARAMETERS DESCRIBED BY POSSIBILITY DISTRIBUTIONS}

In this section, we describe the process used to update, in a Bayesian framework, the epistemicallyuncertain parameters of aleatory models by means of real data (i.e., in this case, the uncertain probabilities of the events in Fault and Event Tree models). The process includes the following steps:

- Build the belief and plausibility functions of the parameters according to the a priori available information (e.g., industry data or experts opinions).

- Transform the prior belief and plausibility functions into prior possibility distribution.

- Update the prior possibility distribution using plant specific data in order to obtain the corresponding posterior possibility distribution.

- Transfer the posterior possibility distribution to belief and plausibility functions.

\subsection{Build the belief and plausibility functions}

In general, the data bank used in PRA industry (such as NUREG/CR reports) provides the mean, the 5-th and 95-th percentiles of the distributions of the epistemically-uncertain parameters (i.e., the basic events probabilities). We can treat 5-th and 95-th percentiles as the lower and upper bounds, respectively, of the uncertain parameter range. Let $m, M$ and $\mu$ be the minimum, maximum and mean values, respectively. The upper bound on probability (plausibility function) over this range can be found by determining the largest possible values attained by a distribution function under specified constraints (Ferson S. et al. 2003). Consider an arbitrary value $x$ $\in[m, \mu]$, let the value $p$ of a distribution at $x$ represent the probability mass at and to the left of $x$. However, the mass must be balanced by mass on the 
right of the mean. The greatest possible mass would be balanced by assuming that the rest of the probability, $1-p$, is concentrated at $M$. Likewise, the arrangement of mass on the left side requires the least balance when it is all concentrated at the point $x$. These considerations lead to the expression $p x+$ $(1-p) \mathrm{M}=\mu$ which can be solved to yield $p=(M-$ u) / $(M-x)$, specifying the largest value of the distribution function for the value $x$. If there were any more probability mass at values less than or equal to $x$, the constraint of the mean could not be satisfied by any arrangement of mass at values less than or equal to $M$. Clearly, its distribution function is one for $x \in[\mu, M]$.

The lower bound on probability (belief function) over this range can be derived by similar arguments. We can yield the lower bound distribution function is zero for $x \in[m, \mu]$ and $p=(x-u) /(x-m)$ for $x$ $\in[\mu, M]$.

\subsection{Prior possibility distributions}

Once we build the belief and plausibility functions, we can change them to a prior possibility distribution such that $\pi(x)=(M-u) /(M-x)$ for $x$ $\in[m, \mu]$ and $\pi(x)=1-(x-u) /(x-m)$ for $x \in[\mu$, $M]$, as we discussed in subsection 2.3. The prior possibility distribution is a unimodal continuous distribution with core $\{u\}$.

\subsection{Purely possibilistic Bayes theorem: posterior possibility distributions}

In probability theory, the Bayes' rule can be stated as follows. Let $X$ be the epistemically-uncertain parameter of the aleatory model $f(y \mid x)$ of the generic random variable $Y$ and $g(x)$ be the prior probability density function of $X$. For example, in the risk assessment context of this paper $Y$ may represent a binary variable taking value 1 , if a given component of the NPP of interest fails to perform its mission, or 0 if it succeeds (in other words, the aleatory model $f(y \mid x)$ is described by a Bernoulli distribution); correspondingly, $X$ represents the parameter of the Bernoulli distribution, i.e., in this case, the probability of failure of the NPP component. Moreover, let $y$ represent the new information/evidence available for the analysis: referring to the example above, $y$ may be represented the number $n$ of failures of a NPP component (type) of interest over a given number $N$ of demands. The objective of the classical, purely probabilistic Bayesian analysis is to update the a priori representation $g(x)$ of $X$ on the basis of the new evidence acquired, i.e., to calculate the posterior probability distribution $g(x \mid y)$ of $X$ after $y$ is obtained, as follows:

$$
g(x \mid y)=\frac{L(x \mid y) g(x)}{\int L(x \mid y) g(x) d x}
$$

where $L(x \mid y)$ is the likelihood of parameter $X=x$ given the new information/evidence available $(L(x \mid y)$ is obviously proportional to the probability of obtaining the experimental evidence $y$ given that $X=$ $x$, i.e., $L(x \mid y) \sim f(y \mid x)$ ). The posterior probability density function $g(x \mid y)$ combines the prior information $g(x)$ with new collected information represented by $L(x \mid y)$, which models the relationship between $X$ and $Y$.

In this paper the prior information on $X$ is represented by a possibility distribution $\pi(x)$, so the objective of our analysis is to calculate the posterior possibility distribution $\pi(x \mid y)$ of $X$ after $y$ is obtained. To this aim, we employ a method based on a purely possibilistic counterpart of the classical, probabilistic Bayes' theorem (Lapointe \& Bobée 2000):

$$
\pi(x \mid y)=\frac{\pi^{L}(x \mid y) \pi(x)}{\max _{x}\left[\pi^{L}(x \mid y) \pi(x)\right]}
$$

where $\pi^{L}(x \mid y)$ is the possibilistic likelihood of the parameter $X$ given the newly observed data $y$, and quantities $\pi(x \mid y)$ and $\pi(x)$ are defined above. Notice that $\left\{\max _{x}\left[\pi^{L}(x \mid y) \pi(x)\right]\right\}$ is a normalization factor such that $\{\max [\pi(x \mid y)]\}=1$, as required by possibility theory (Baudrit \& Dubois 2006).

It is worth mentioning that forms of the possibilistic Bayes' theorem alternative to (12) can be constructed as a result of other definitions of the operation of 'conditioning' with possibility distributions: the reader is referred to (Lapointe \& Bobée 2000) for technical details. In this paper, expression (12) has been chosen because "it satisfies desirable properties of the revision process and lead to continuous posterior distributions" (Lapointe \& Bobée 2000).

The possibilistic likelihood $\pi^{L}(x \mid y)$ is here obtained by transforming the classical probabilistic likelihood function $L(x \mid y)$ through normalization, i.e., $\pi^{L}(x \mid y)=L(x \mid y) / \sup \{L(x \mid y)\}$. This choice has been made for the following main reasons: (i) the transformation is simple and can be straightforwardly applied to any distribution; (ii) the resulting possibilistic likelihood is very closely related to the classical, purely probabilistic one (which is theoretically well-grounded) by means of the simple and direct operation of normalization that preserves the 
"original structure" of the experimental evidence; (iii) it can be easily verified that the resulting possibilistic likelihood keeps the sequential nature of the updating procedure typical of the standard Bayes' theorem. On the other hand, it has to be also admitted that the resulting possibility distributions do not in general adhere to the probability-possibility consistency principle (Baudrit \& Dubois 2006).

It is worth noting that other techniques of transformation of probability density functions into possibility distributions exist, but the corresponding details are not given here for brevity sake: the interested reader is referred to (Flage et al. 2013) for some proposed techniques, e.g., the principle of maximum specificity and the principle of minimal commitment. Also, it has to be noticed that other techniques are available to construct possibility distributions (and, thus, possibilistic likelihood functions) directly from rough experimental data (i.e., without resorting to probability-possibility transformations): see, e.g., (Serrurier \& Prade 2011).

\subsection{Updated belief and plausibility functions}

The posterior possibility distribution $\pi(x \mid y)$ thereby obtained is a unimodal continuous distribution with (new) core $\{a\}$. Then, we can obtain the corresponding belief and plausibility functions using equations (9) and (10).

\section{CASE STUDY}

We used a simplified small loss of coolant accident (SLOCA) as the case study to demonstrate the Bayesian updating process. The system layout of the case study is shown in Figure 1. After a SLOCA, the core inventory falls down to certain level and triggers the higher pressure injection system to pump the water to make up the water; if the high pressure injection system fails, the reactor operators have to depressurize manually to make the low pressure injection system overcome the core pressure to pump the water into the reactor pressure vessel. Even if the high pressure injection system works properly, the low pressure system still has to fulfill the long term heat removal function to bring the reactor to cold shutdown condition. The SLOCA event tree is shown in Figure 2.

The heading HP in the SLOCA event tree representing the high pressure injection system, has to open two air-operated valves (AOV) and start the injection pump when receiving the auto-start signal. Any component failing by demand and pump failing while running imply the high pressure injection function failure. The fault tree of the HP is shown in Figure 3. There are four basic events in the HP fault tree.

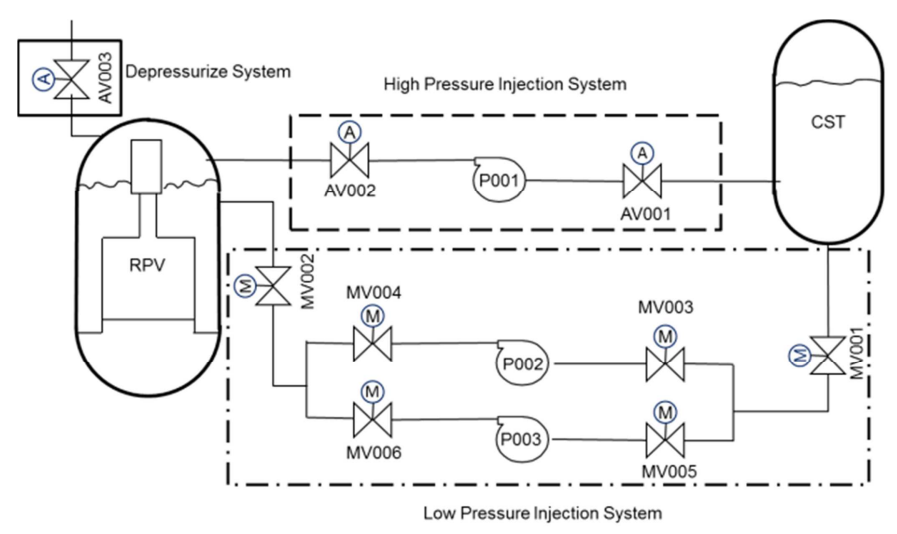

Figure 1. Simplified system layout of case study

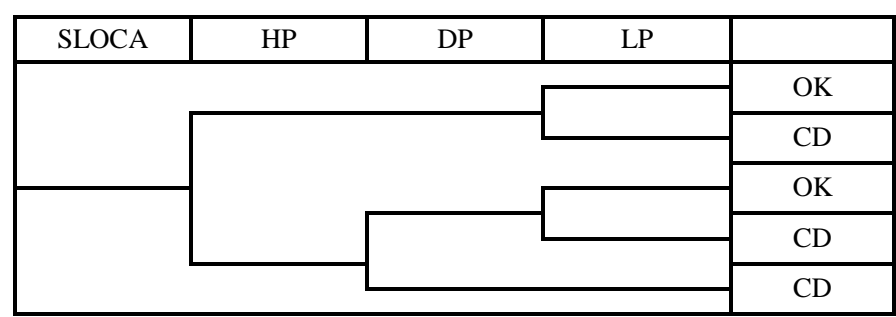

Figure 2. Small loss of coolant accident (SLOCA) event tree

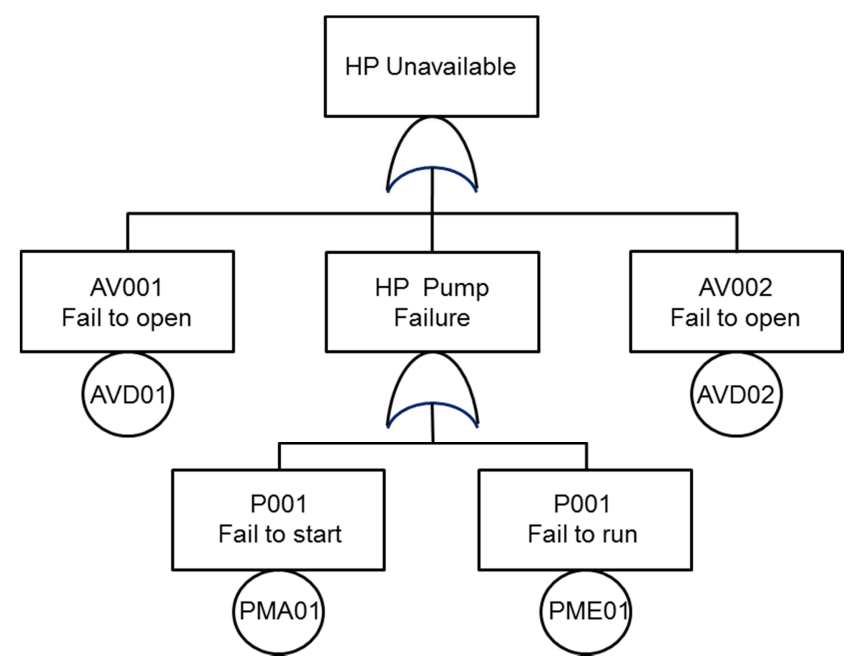

Figure 3. The fault tree of heading HP

In case of high pressure injection function failure, the reactor operators have to depressurize the core manually. The fault tree of heading DP is shown in Figure 4: the depressurization system includes only two basic events, a human action and a mechanical fault.

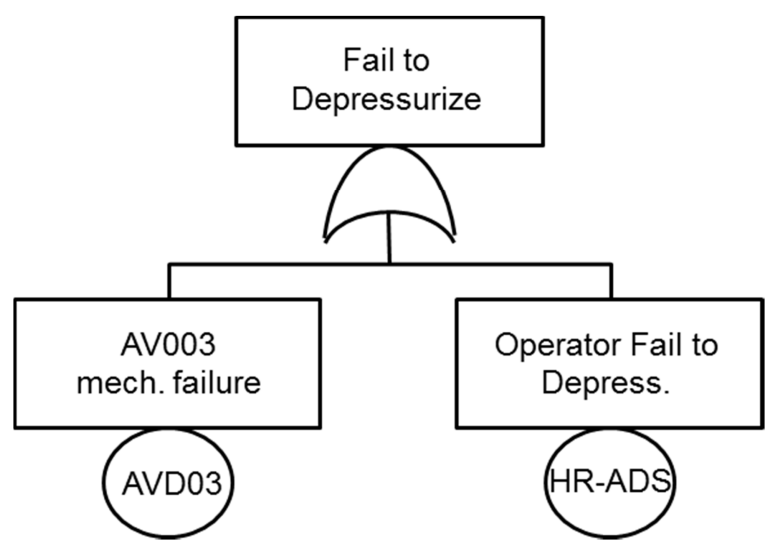

Figure 4. The fault tree of heading DP 
From the system layout, there are two trains in the low pressure injection system and they have common suction and injection valves. Each train can provide $100 \%$ demand flow under SLOCA condition. We did not consider any common failure between components. The fault tree of LP is shown in Figure 5. There are ten basic events in LP fault tree, including six motor operated valves (MOV) fail to open, two pumps fail to start and two pumps fail while running. The probabilities of all these basic events are epistemically-uncertain.

The industry data we use to build the prior possibility distributions for the basic events probabilities (see Section 3.1 and 3.2) are from NRC report NUREG/CR-6928 (Eide S.A. et al. 2007) (Table 1); the plant specific data used to perform the Bayesian update are obtained from a nuclear power plant with 2 BWR6 units which were commercially operated for about 25 years (Table 1). The data for basic events shown in Table 1 is also used in their PRA report.

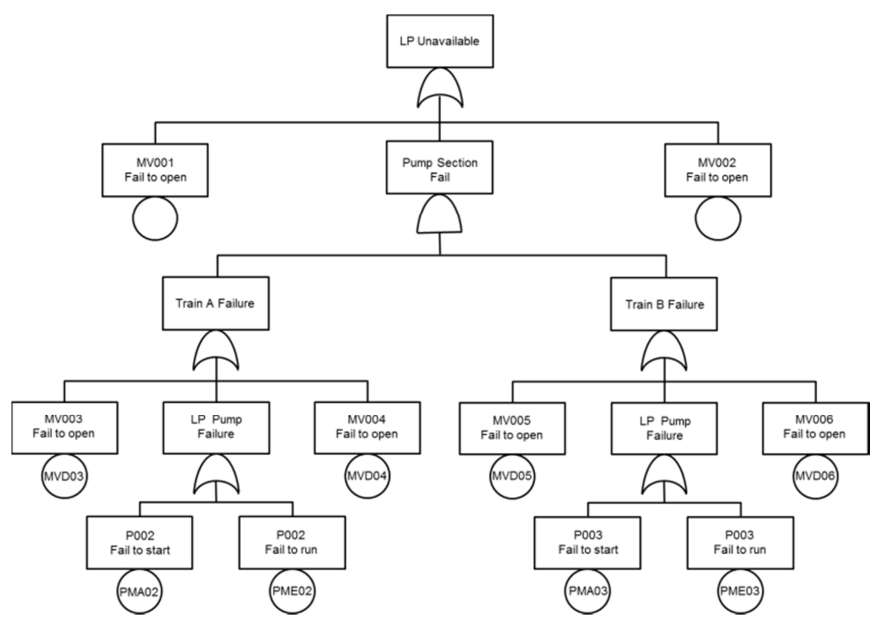

Figure 5. The fault tree of heading LP

Table 1. Data used for the basic events probabilities

\begin{tabular}{|c|c|c|c|c|c|}
\hline \multirow{2}{*}{$\begin{array}{l}\text { Basic } \\
\text { Event }\end{array}$} & \multicolumn{3}{|c|}{ Industry Data } & \multicolumn{2}{|c|}{ Specific Data ${ }^{*}$} \\
\hline & $5 \%$ & mean, & $95 \%$ & $\begin{array}{c}\text { \# of } \\
\text { failure, } n\end{array}$ & $\begin{array}{l}\text { \# of demand, } N \\
\text { (running hours, } T \text { ) }\end{array}$ \\
\hline AVD & $6.0 \mathrm{E}-5$ & $1.2 \mathrm{E}-3$ & $4.0 \mathrm{E}-3$ & 7 & 8844 \\
\hline MVD & $8.0 \mathrm{E}-5$ & $1.0 \mathrm{E}-3$ & $3.0 \mathrm{E}-3$ & 9 & 9052 \\
\hline PMA & $6.0 \mathrm{E}-5$ & $1.5 \mathrm{E}-3$ & $5.0 \mathrm{E}-3$ & 9 & 4538 \\
\hline PME & $5.0 \mathrm{E}-5$ & $4.0 \mathrm{E}-4$ & $1.0 \mathrm{E}-3$ & 2 & $3329.8 \mathrm{~h}$ \\
\hline HR-ADS ${ }^{*}$ & $1.3 \mathrm{E}-4$ & $3.4 \mathrm{E}-3$ & $1.3 \mathrm{E}-2$ & -- & -- \\
\hline
\end{tabular}

* Data from plant HRA report

First, we used the industry generic data to build the prior belief and plausibility functions for each basic event probability (Section 3.1). The 5-th and 95-th percentiles were set as the lower and upper bounds, respectively, of the uncertain parameter ranges. The resulting belief and plausibility functions are shown in Figure 6 with reference to the AOV only for illustration purposes. The predetermined (i.e., presumed) a priori beta function traditionally used in NPP PRAs to represent the
AOV demand failure probability is also plotted in Figure 6 for comparison.

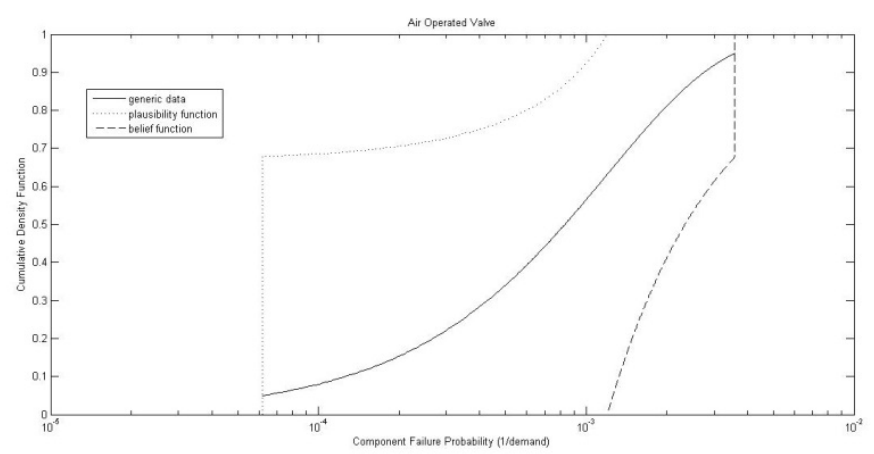

Figure 6. The belief and plausibility functions for the AOV failure probability

Second, we transform the belief and plausibility functions into possibility distributions (Section 3.2). This distribution is the prior possibility distribution used in the Bayesian update. As shown in Figure 7 with reference to the AOV failure probability, the left part of prior possibility distribution coincides with the corresponding plausibility function whereas the right part is equal to the complement of the corresponding belief function.

Then, the prior possibility distribution is revised using the plant specific data (Section 3.3). Notice again that the possibilistic likelihood functions used in the possibilistic Bayes theorem (12) are obtained by normalization of the corresponding probabilistic functions: for example, (normalized) Binominal distributions are used to update the (uncertain) probabilities of AVDs, MVDs and PMAs, whereas (normalized) Poisson distributions are employed to update the rates of PMEs. The posterior possibility distribution of the AOV failure probability is shown in Figure 7 for comparison. It can be seen that after Bayesian update, the distribution is more peaked than before.

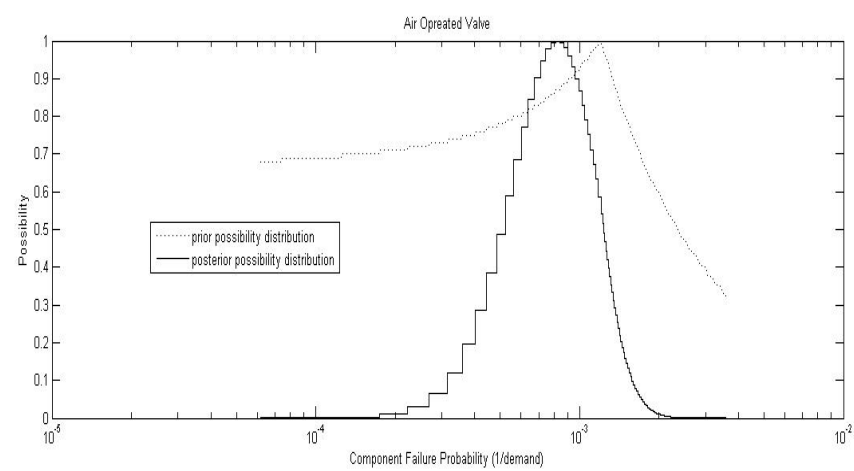

Figure 7. Possibility distributions for the AOV failure probability

For uncertainty propagation purposes, we reconvert the posterior possibility distribution of each basic event to belief and plausible functions (Section 2.3). Figure 8 shows the prior and posterior belief 
and plausibility functions for the AOV failure probability. In addition, for comparison purposes Figure 8 also reports the posterior distribution for the AOV failure probability obtained by a classical, purely probabilistic Bayesian analysis employing as a prior the predetermined (i.e., presumed) beta function of Figure 6: it can be seen that the resulting posterior distribution is well bounded by the posterior belief and plausibility functions (Figure 8).

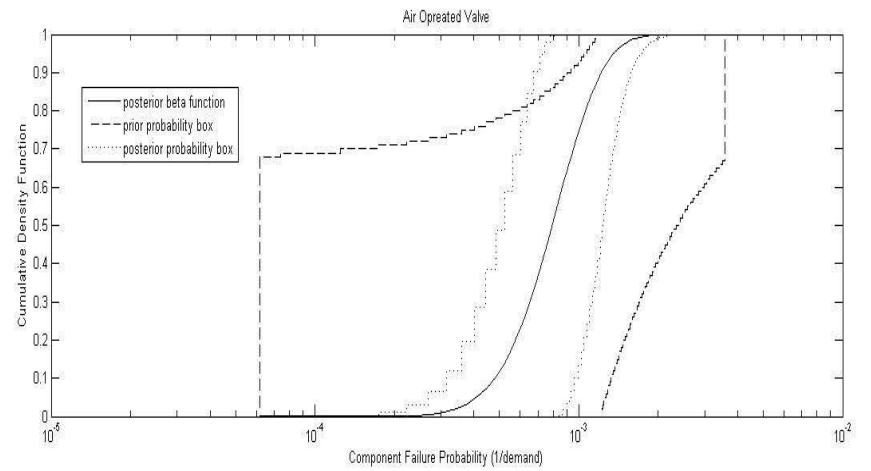

Figure 8. The prior and posterior probability bounds for the AOV failure probability

Finally, after the Bayesian update process, the uncertainty in the basic event probabilities (i.e., the inputs of the analysis) is propagated by Monte Carlo simulation to the Conditional Core Damage Probability (CCDP) (i.e., the output of the analysis). The Monte Carlo uncertainty propagation for the SLOCA CCDP was performed and the results are shown in Figure 9. In addition, for comparison purposes Figure 9 also reports the prior and posterior cumulative distributions for the CCDP obtained within a classical, purely probabilistic Bayesian analysis in which the basic events probabilities are described by beta functions with characteristics (i.e., mean, 5-th and 95-th percentiles) given in Table 1. The traditional Monte Carlo uncertainty analysis results are well bounded by those obtained by the possibilistic approach.

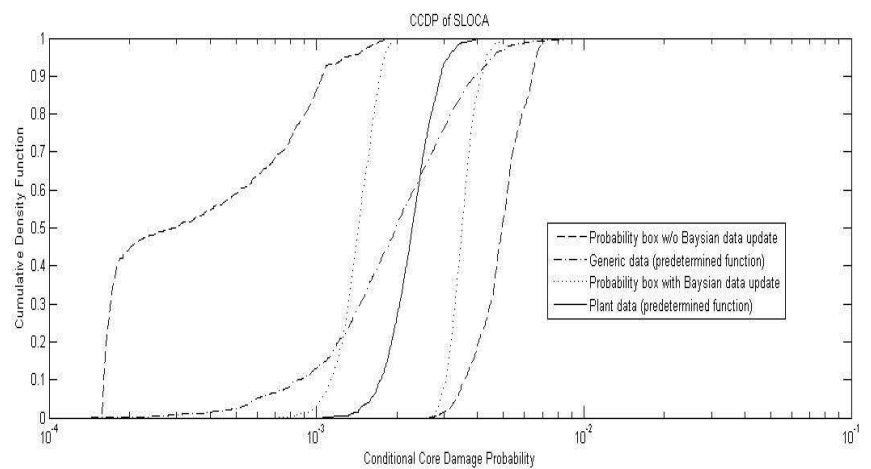

Figure 9. The prior and posterior probability bounds for the CCDP

The area between the belief and plausibility functions represents the uncertainty bound: the bigger value means less information to make a decision. In this study case, the area between the posterior functions reduces to about $48.5 \%$ of that between prior functions. Additionally, the $95 \%$ upper bound value to $5 \%$ lower bound value ratio also reduces from 41.7 to 4.0. This means that we have more confidence to make a decision when comparing to a threshold criterion.

\section{CONCLUSION}

In the context of NPP PRA, the traditional decision making process within the probabilistic approach relies on the comparison of risk indicators to a safety threshold. The uncertainty bound of the risk indicators usually makes it difficult to make a decision. Many recent studies concluded that it is more appropriate to use a family of probability distributions for representing imprecise and incomplete information rather than a unique presumed probability distribution. Although using belief and plausibility functions for uncertainty analysis provides more realistic and robust results, it expands the uncertainty bound of risk indicators.

For a single probability density function, the Bayes' rule is commonly used to reduce the uncertainty bounds when new information such as plant specific data is available. We have shown that the Bayes' rule can also be applied to revise a family of probability distributions. In this paper, we have built the process and demonstrate it by a simplified SLOCA case study. The final result shows that the Bayesian update can reduce the uncertainty bound significantly and will be very helpful to decision making.

\section{REFERENCES}

Baudrit C, Couso I, Dubois D. 2007. Joint propagation of probability and possibility in risk analysis: Toward a formal framework. International Journal of Approximate Reasoning 2007; 45:82-105.

Baudrit, C. \& Dubois, D. 2006. Practical Representations of Incomplete Probabilistic Knowledge. Computational Statistics \& Data Analysis 51(1): 86108.

Dempster A. 1967. Upper and lower probabilities induced by a multivalued mapping. Annuals of Mathematical Statistics 1967; 38:325-39

Drouin M, Parry G, Lehner J, Martinez-Guridi G, LaChance J, Wheeler T. 2010. Guidance on the treatment of uncertainties associated with PRA in risk-informed decision making. Technical report NUREG 1855-V.1; U.S Nuclear Regulatory Commission; 2010.

Dubois D., Nguyen H.T., Prade H., 2000. Possibility theory, probability and fuzzy sets: misunderstanding, bridges and gaps. Fundamentals of Fuzzy Sets. Kluwer, Boston, MA, pp.343-438. 
Dubois D., Prade H., 1992. When upper probabilities are possibility measures. Fuzzy Sets and Systems 49. 6574.

Durga Rao K, Kushwaha H, Verna A, Srividya A. 2007. Quantification of epistemic and aleatory uncertainties in level-1 probabilistic safety assessment studies. Reliability Engineering and System Safety 2007; 92:947-56

Eide S.A., Wierman T.E., Gentillon C.D., Rasmuson D.M., Atwood C.L. 2007. Industry-Average Performance for Components and Initiating Events at U.S. Commercial Nuclear Power Plant. NUREG/CR6928. U.S. Nuclear Regulatory Commission Office of Nuclear Regulatory Research

Ferson S., Kreinovich V., Ginzburg L., Myers D.S., Sentz K., 2003. Constructing Probability Boxes and Dempster-Shafer Structures. SAND REPORT SAND2002-4015, Sandia National Laboratories 2003

Flage, R., Baraldi, P., Zio, E., Aven, T. 2013. Probability and possibility-based representations of uncertainty in fault tree analysis. Risk Analysis 33(1): 121-33.

Lapointe S. \& Bobeè B., 2000. Revision of possibility distributions: A Bayesian inference pattern. Fuzzy Sets and Systems 116: 119-140.

Le Duy T.D, Dieulle L, Vasseur D, Berenguer C, Couplet M. 2013. An alternative framework using belief functions for parameter and model uncertainty analysis in nuclear probabilistic risk assessment applications. Proceedings of the Institution of Mechanical Engineers, Journal of Risk and Reliability.

Serrurier, M. \& Prade, H., 2011. Maximum-Likelihood Principle For Possibility Distributions Viewed As Families Of Probabilities. 2011 IEEE International Conference on Fuzzy Systems June 27-30, 2011, Taipei, Taiwan: 29872993.

Shafer G. 1976. A Mathematical Theory of Evidence. Princeton University Press; 1976

Walley P. 1991. Statistical Reasoning with Imprecise Probabilities. Chapman \& Hall, London; 1991 